\title{
Descripción de la producción de etanol a partir de desechos orgánicos domésticos y hojarasca, utilizando modelos matemáticos aplicados en el software Derive 6.1 y la hoja de cálculo Excel ${ }^{1}$
}

\author{
Description of the production of ethanol from organic \\ household waste and litter, using mathematical models applied \\ in the software Derive 6.1 and Excel spreadsheet \\ Descrição da produção de etanol a partir de resíduos \\ domésticos orgânicos e lixo, utilizando modelos matemáticos \\ aplicados no software Derive 6.1 e planilha Excel
}

Recibido: mayo de 2013

Aceptado: agosto de 2013
Jhon Franklin Espinosa Castro ${ }^{2}$

Helen Tatiana Hernández Jáuregui ${ }^{3}$

Luz Francy Yáñez Meneses ${ }^{4}$

\section{Resumen}

La presente propuesta tiene como objetivo describir la producción de etanol a partir de desechos orgánicos domésticos y hojarasca, utilizando modelos matemáticos aplicados en los programas Derive 6.1 y Excel; en procesos químicos, biológicos, físicos y matemáticos en el desarrollo analítico - gráfico, para la optimización de tiempo, y la verificación de las etapas del procedimiento. De acuerdo a lo anterior, la metodología esta basada en un enfoque cuantitativo de tipo descriptivo, debido a que se harán explicaciones coherentes en cada etapa. Así, esta propuesta da inicio a ideales investigativos, avances a una cultura ecológica en la protección y conservación de la misma, empleando como alternativa el uso de biocombustibles.

Palabras clave: Residuos; fermentación; etanol; abono; modelo; recursos informáticos; software; matemáticas escolares; cálculo; derivación.

\begin{abstract} the use of biofuels.
\end{abstract}

This proposal aims to describe the production of ethanol from organic household waste and litter, using mathematical models applied in Derive 6.1 and Excel programs, in chemical, biological, physical and mathematical analytical development - graphic, for optimization of time, and verification of the method steps. According to the above, the methodology is based on a quantitative, descriptive, because it will make coherent explanations at each stage. Thus, this proposed research ideal kicks, advances to an ecological culture in the protection and preservation of the same, using alternatively

\footnotetext{
1 Artículo de Investigación

2 Universidad Nacional Experimental del Táchira. Contacto: jhon_franklin_espinosa@hotmail.com,

3 Universidad Francisco de Paula Santander. Contacto: helen02092@gmail.com

4 Universidad Francisco de Paula Santander. Contacto: viomubioluz@gmail.com
} 
Keywords: waste, fermentation, ethanol, fertilizer, model, computing resources, software, school mathematics, calculation, derivation.

\section{Resumo}

Esta proposta tem como objetivo descrever a produção de etanol a partir de resíduos domésticos orgânicos e lixo, utilizando modelos matemáticos aplicados em Derive 6.1 e programas Excel, na indústria química, desenvolvimento analítico físico e matemático biológico - gráfico, para Optimização do tempo, e a verificação dos passos do método. De acordo com o exposto, a metodologia baseia-se num quantitativo, descritivo, porque ele vai fazer explicações coerentes, em cada fase. Assim, esta pesquisa proposto chutes ideais, os avanços para uma cultura ecológica na proteção e preservação do mesmo, usando alternativamente, a utilização de biocombustíveis.

Palavras-chave: resíduos, a fermentação, o etanol, fertilizantes, modelo, os recursos de computação, software, matemática escolar, de cálculo, de derivação.

\section{Introducción}

Actualmente cada escenario viviente está constituido por materiales orgánicos e inorgánicos que a su vez conllevan a cadenas repetitivas; no obstante el ser humano se encuentra en la constante búsqueda del mejoramiento y aseguramiento de su bienestar. Así mismo, uno de los ámbitos comprendidos en el mundo actual es la producción de energías renovables que en su estructura no representa delimitación ni peligro biológico, comparándolo con los combustibles derivados del petróleo, grandes precursores del calentamiento global. Si partimos de la acogida que han tenido los biocombustibles en los últimos años, es necesario explorar nuevas materias primas adecuadas que proporcionen altos rendimientos y por consiguiente sostenibilidad. Por lo tanto, la presente propuesta comprende la conversión del material lignocelulosico y residuos orgánicos domésticos en glucosa, a través de fermentación alcohólica para la producción de etanol en un biorreactor.

\section{Materiales y métodos}

Es necesario considerar el uso de recipientes, triturador, biorreactor, destilador, termómetros, pH-metro, computador (análisis y control), materia prima (almidón, fructosa y lignocelulosa).
Además, se implementa una metodología cuantitativa, enfatizada en un tipo de investigación descriptiva, debido a que, cada etapa en la producción de etanol esta especificada, a través de mapas conceptuales, tablas, imágenes, cálculos y gráficas. Igualmente, se trabajará con un diseño de experimentos, para analizar diferencias significativas de los residuos que más aportan glucosa como sustrato básico para llevar a cabo el proceso de la fermentación. Por último, para el proceso matemático, gráfico y la presentación de datos, se utilizarán los programas Derive 6.1 y Excel.

\section{Resultados y discusión}

En la obtención de los productos finales se debe tener en cuenta un biorreactor en proceso discontinuo y continuo. A continuación, se presenta la imagen del biorreactor que se utilizará:

La digestión ocurre en la parte inferior, y en la parte superior se acumulan gases. El tiempo de mezcla $\left(t_{m}\right)$ depende del grado de homogeneidad deseado, y se realiza para eliminar las capas que suelen formarse en la parte superior. Ahora, se presentan las etapas de la producción de etanol:

Es indispensable realizar una filtración al vacío para separar el bagazo del líquido residual. Este último, al contener etanol inmerso en agua, forma una mezcla miscible, siendo necesario 


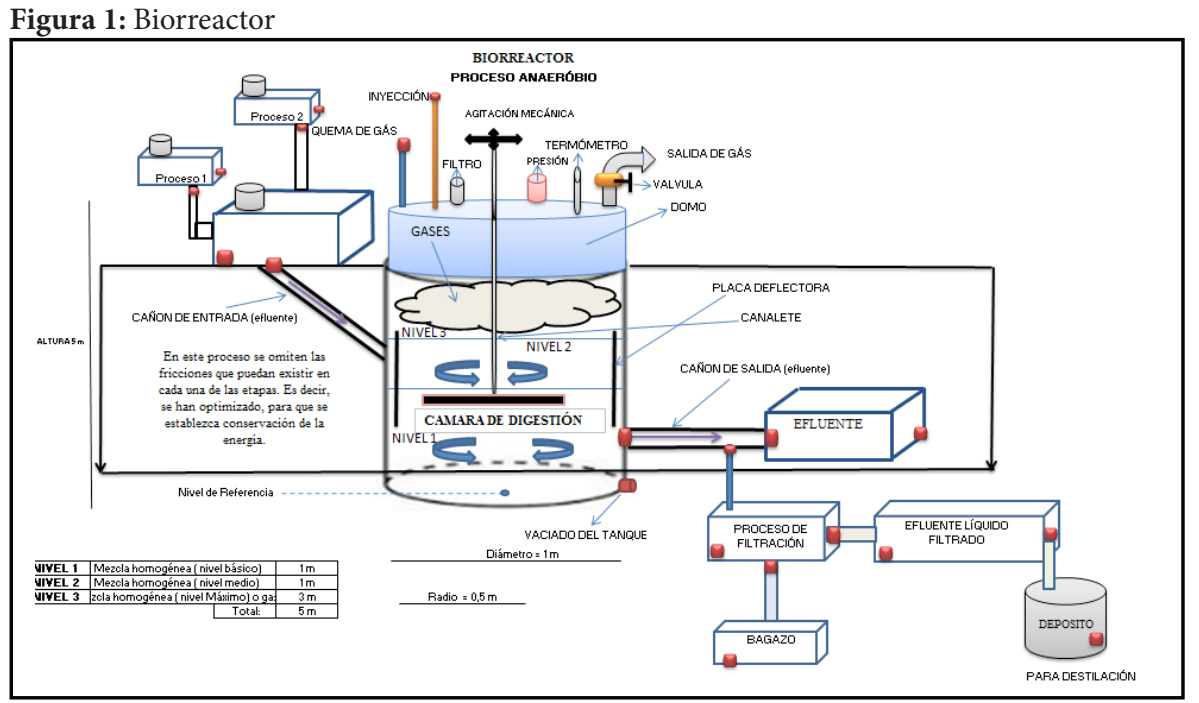

Fuente: Elaboración propia

Figura 2. etapas de la producción de etanol

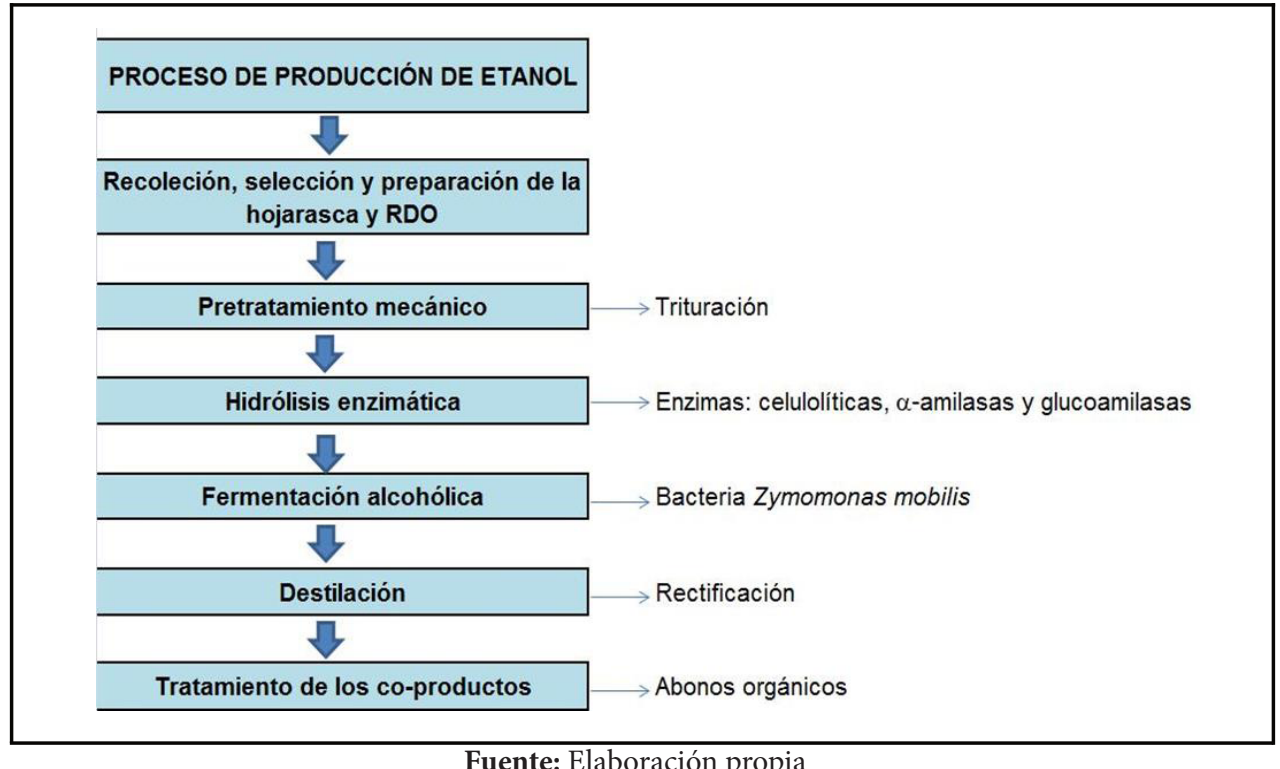

Fuente: Elaboración propia

realizar una destilación para la respectiva separación. Así, por un proceso de rectificación se lleva cabo el diseño de la columna de destilación con el número de platos necesarios para alcanzar la concentración de etanol deseada.

La ecuación diferencial asociada al problema de vaciado del tanque con geometría de un cilindro es $\mathrm{A}(\mathrm{h}) \mathrm{dh} / \mathrm{dt}=-\mathrm{ac} \sqrt{ } 2 \mathrm{gh}$; cuando existe aporte de líquido al tanque, y al mismo tiempo esta saliendo por el orificio, la ecuación diferencial es $A(h) d h / d t=Q-a c \sqrt{ } 2 g h$. La altura del efluente en cualquier instante de tiempo t, en base a las dimensiones del biorreactor es $\mathrm{h}(\mathrm{t})=((-7 \mathrm{t}+10 \sqrt{ } 10$ $\sqrt{200}) /(10 \sqrt{10}))^{\wedge} 2$. El caudal se establece por $\mathrm{c}=\mathrm{vA}=\sqrt{ } 2 \mathrm{gh}^{\star} \pi \mathrm{r}^{\wedge} 2$. Por otra parte, el crecimiento de la población de microorganismos, se enfrenta a factores que impiden el crecimiento con el tiempo, debido a la escasez de alimentos o de agua, y está dado por la ecuación diferencial de crecimiento limitado. dy/dt=kM-ky (García, J.; López, G. 2011, p. 3). Donde, $\mathrm{M}=$ tamaño de la población máxima; $\mathrm{y}=$ tamaño de la población normal; $\mathrm{k}=$ razón de crecimiento. Luego, el modelo matemático en un instante de tiempo $t$, en un intervalo dado es $y=-$ $\mathrm{ce}^{\wedge}(-\mathrm{kt})+\mathrm{M}$. Con $\mathrm{c}=$ constante de integración $\mathrm{y} \mathrm{e}=$ 
constante Euler. Ambas modelos son resueltos por el programa Derive 6.1, la aplicación de la sintaxis $y$ las condiciones iniciales es SOLVE (SEPARABLE $\left(\mathrm{p}(\mathrm{x}), \mathrm{q}(\mathrm{y}), \mathrm{x}, \mathrm{y}, \mathrm{x} \_0, \mathrm{y} \_0\right), \mathrm{y}$, Real) (Sánchez, et al, 2001, p. 225).

La ecuación general del balance de materia dentro del proceso del biorreactor en discontinuo y continuo es dm/dt=q_1+r_1-q_2-r_2 (Doran, 1998, p. 115). Donde, $m$ es la suma total de la cantidad balanceada en el sistema, y los cuatro términos restantes son velocidades que pueden variar en fun-

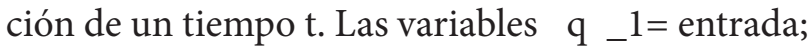
q_2 = salida; r_1 = generación y r_2 = consumo. Es decir, acumulación $=$ entrada + generación - salida - consumo. Dependiendo del bioproceso, la ecuación presenta una variación. Luego, para el proceso discontinuo, donde no existe flujo de entrada ni de salida en el biorreactor, el tiempo necesario para el consumo de toda la masa del sustrato por parte del microorganismo, está dado por la ecuación ds/ $\mathrm{dt}=-\left(\mu \_\mathrm{max} / \mathrm{Y} \_\mathrm{xs}+\mathrm{q} \_\mathrm{p} / \mathrm{Y} \_\mathrm{PS}+\mathrm{m} \_\mathrm{s}\right) \mathrm{x} \_0 \mathrm{e}^{\wedge}\left(\mu \_\right.$ $\max \mathrm{t}$ ) (Doran, 1998, p. 375). Donde, $\mu \_\max$ es la velocidad específica máxima de crecimiento, Y_xs el rendimiento verdadero de biomasa a partir de sustrato, q_p la velocidad especifica de formación de producto, Y_ps el rendimiento verdadero de producto a partir de sustrato y m_s el coeficiente de mantenimiento. Si todos los términos situados entre paréntesis son constantes durante el cultivo, la anterior ecuación puede integrarse directamente con la condición inicial $s=s 0$ a $t=0$, para obtener la siguiente ecuación: $t \_b=1 / \mu \_\max \ln \left[1+\left(s \_0\right.\right.$ $\left.s \_f\right) /\left(\left(1 / Y \_x s+q \_p /\left(\mu \_(\max )\right.\right.\right.$ Y_PS $)+m_{-} s / \mu \_$ max ) x_0 )] donde t_b es el tiempo de cultivo en discontinuo y s_f la concentración final del sustrato. La densidad de células que se puede alcanzar a partir del tiempo t_b está dada por la ecuación $\mathrm{dx} / \mathrm{dt}=\left(\mu-\mathrm{k} \_\mathrm{d}\right) \mathrm{x}$ donde $\mu$ es la velocidad específica de crecimiento y k_d es la constante específica de muerte celular. Debido a que, en un cultivo discontinuo permanece casi constante e igual ha $\mu \_\max y$ $\mathrm{k} \_\mathrm{d}$ permanece igualmente constante, la anterior ecuación puede integrar directamente obteniendo una relación entre el tiempo de operación discontinuo y la concentración de células, y si además, la velocidad de muerte celular es despreciable en comparación con la del crecimiento, tenemos $x=x \_(0) e^{\wedge}\left(\mu \_m a x t\right)$. Para el proceso continuo, los parámetros de operación característicos son la velocidad de dilución $\mathrm{D}$ y el tiempo medio de residencia $\tau$. Estos parámetros están relacionados de la siguiente manera $\tau=1 / \mathrm{D}=\mathrm{V} / \mathrm{F}($ Doran, 1998, p. 381). Donde, D viene definido por la ecuación $\mathrm{D}=\mathrm{F} / \mathrm{V}$. Para el presente proceso, los balances de materia se encuentran en estado estacionario. Así, la ecuación en estado estacionario para la biomasa es: $\mathrm{Fxi}-\mathrm{Fx}+\mu \mathrm{xV}-\mathrm{kdxV}=0$, obteniéndose que $\mu=\mathrm{D}$. Por lo tanto, sustituyendo $\mu \nabla \mathrm{D}$ en la ecuación de Monod se obtiene $\mathrm{D}=\left(\begin{array}{l}\mu \_\max \\ \mathrm{s}\end{array}\right)$ / (k_s+s) donde k_(s ) es la constante de sustrato. Reordenando la ecuación se da una expresión para la concentración de sustrato en función de la velocidad de dilución $s=($ Dk_s $) /\left(\mu \_m a x-D\right)$. La ecuación de sustrato limitante en estado estacionario viene dada por Fsi - Fs - $\left(\mu / Y \_x s+q \_p / Y \_P S\right.$ $\left.+\mathrm{m} \_\mathrm{s}\right) \mathrm{xV}=0$. Ahora, la siguiente ecuación para expresar la concentración de células en estado estacionario $\mathrm{x}=\mathrm{D}\left(\mathrm{s} \_\mathrm{i}-\mathrm{s}\right) /\left(\mathrm{D} / \mathrm{Y} \_\mathrm{XS}+\mathrm{q} \_\mathrm{p} / \mathrm{Y} \_\mathrm{PS}+\mathrm{m} \_\mathrm{s}\right.$ ). Luego, puede aplicarse la ecuación del balance de materia en estado estacionario al producto de fermentación encontrando Fpi $-\mathrm{Fp}+\mathrm{qpxV}=0$, donde q_p es la velocidad especifica de formación de cualquier clase de producto. Resolviendo esta ecuación, se obtiene una expresión para la concentración del producto en estado estacionario en función de la concentración de biomasa $\mathrm{x}$ : $\mathrm{p}=\mathrm{pi}+$ (q_p $x$ )/D. Igualmente, el resultado se establece porque se utiliza el programa Derive $6.1 \mathrm{y}$ Excel.

\section{Agradecimientos}

A Llary Laudith Navarro y Harvey Eduardo Hernández.

\section{Conclusiones}

Al hacerse realidad esta propuesta unificaría un conjunto de conocimientos en diferentes áreas y aportaría al equilibrio de la naturaleza generando un biocombustible para diversas actividades. Además del etanol, los co - productos obtenidos (bagazo y vinaza) proporcionan nutrientes para 
enriquecer el suelo. Por último, la implementación de programas matemáticos en procesos investigativos, permite optimizar tiempo, analizar el desarrollo analítico, gráfico y validar datos.

\section{Referencias}

Doran, P. (1998). Principios de ingeniería de los bioprocesos. Editorial Acribia. Zaragoza, España, p. 115, 375,381 .
García, J.; López, G. (2011). Modelado del Crecimiento de Bacterias al Interior de un Biodigestor. Cap\&Cua, 5(1), p. 3 - 4.

Sánchez, L.; Legua, M.; Moraño, J. (2001). Matemáticas con Derive. Editorial Universidad Politécnica de Valencia. Departamento de matemáticas aplicada. 1a edición. p. 225 - 226, $233-234$. 